\title{
Genre de discours et technologie discursive
}

Tweet, twittécriture et twittérature

\section{Marie-Anne Paveau}

\section{(2) OpenEdition}

Journals

Édition électronique

URL : http://journals.openedition.org/pratiques/3533

DOI : $10.4000 /$ pratiques.3533

ISSN : 2425-2042

Éditeur

Centre de recherche sur les médiations (CREM)

\section{Édition imprimée}

Date de publication : 1 juin 2013

Pagination : 7-30

\section{Référence électronique}

Marie-Anne Paveau, " Genre de discours et technologie discursive », Pratiques [En ligne], 157-158|

2013, mis en ligne le 18 décembre 2017, consulté le 10 décembre 2020. URL : http://

journals.openedition.org/pratiques/3533; DOI : https://doi.org/10.4000/pratiques.3533 


\title{
Genre de discours et technologie discursive. Tweet, twittécriture et twittérature
}

\author{
Marie-Anne Paveau
}

Université de Paris 13 Sorbonne Paris Cité, Pléiade

\section{Introduction}

La notion de genre est approchée ici sous l'angle du discours : je parlerai de genre de discours et non de genre de texte, de discursivité et non de textualité. Ce choix est celui d'une discussion critique, qui repose sur l'idée que ces notions ne sont pas des catégories discrètes et déterminées. Cette discussion est menée, de visu ou de libro, avec les auteurs qui travaillent la notion de genre depuis les années 1980, depuis qu'elle est entrée dans les champs ${ }^{(1)}$ de la linguistique (voir Branca 1999 pour une synthèse, et les travaux de Adam, Bronckart, Maingueneau, Moirand, Rastier). Parmi ces auteurs, je distingue particulièrement Adam qui est scientifiquement, institutionnellement et éditorialement au centre de ce débat, en particulier via ses deux dernières publications, Adam 2011a et 2011b.

Mon objectif dans cet article est de travailler la notion de genre de discours dans les environnements numériques du web 2.0. Mon objet est constitué par les productions discursives en ligne élaborées par les technologies discursives nécessaires à leur existence. Je préciserai d'abord ma position par rapport à ce qui me semble le logocentrisme des disciplines texte-discours avant de proposer une mise au point sur la notion de genre de discours et la définition que j'en adopte, ainsi que sur les observables discursifs en ligne. Je présenterai ensuite, à partir de l'exemple du réseau de microblogging Twitter, une approche non logocentrée des productions discursives tenant compte de la matérialité des technologies discursives, qui me conduira à la notion de généricité composite des productions du web des réseaux.

(1) Je parle de «champs » au pluriel pour désigner ce que j’appelle en abrégé les disciplines TDI (texte-discours-interaction), c'est-à-dire l'analyse du discours, la linguistique textuelle, l'analyse conversationnelle ou la sociolinguistique, cette dernière ayant intégré à ses outils théoriques une version un peu différente de la notion de genre (voir l'article de Gadet ici-même). 


\section{Texte et contexte dans les disciplines texte-discours}

J'ai deux désaccords avec ce qui me semble être le mainstream autour de la notion de genre, qui repose majoritairement sur une conception encore logocentrée des théories du texte et du discours.

\subsection{Texte et discours}

Le premier concerne le recouvrement de la notion de discours par celle de texte : parler "d' analyse textuelle des discours ", comme le fait Adam depuis l'édition de 2005 de La linguistique textuelle (Adam 2005), me semble présenter le risque de dissoudre la notion de discours, de lui enlever de son épaisseur et surtout de sa matière en le ramenant à une version logocentrée ${ }^{(2)}$. Si le discours et le texte sont bien des produits de deux regards sur les mêmes données empiriques, c'est-àdire des formes langagières en contexte, il n'en reste pas moins que le discours possède des observables spécifiques, dont une analyse textuelle ne peut rendre compte. La notion de constitutivité ${ }^{(3)}$, qui depuis Pêcheux et al. 1971 constitue l'un des traits définitoires de la notion de discours, n'est pas prise en compte par l'approche textualiste.

\subsection{La nature du contexte}

Le second désaccord concerne le contexte, que je trouve insuffisamment pris en compte dans les analyses qui privilégient la notion de genre. Si la « situation » ou « contexte » ou « conditions de production » sont allégués comme déterminants pour les choix ou les contraintes génériques, ces notions restent insuffisantes pour rendre compte de cette constitutivité introuvable : il me semble qu'on peine toujours, dans les disciplines qui étudient les énoncés empiriques, à penser et donc théoriser l'articulation des extérieurs du discours, de nature non langagière, à la matière discursive, de nature langagière. On dira par exemple qu'une situation d'enseignement implique des formes paraphrastiques, explicatives, l'adoption d'un genre de discours didactique, traversé par des formes ordinaires, humoristiques, qui constituent des ruptures appartenant à « la loi du genre». En d'autres termes, si les paramètres sociaux, politiques, historiques, culturels, etc., sont intégrés aux analyses pour rendre compte des modes de formation des discours, ils restent de l'ordre de la contrainte ou de la causalité extérieures : les contextes influent sur la production discursive, la contraignent, la déterminent, voire la colorent. Les analyses restent logocentrées.

Les analyses TDI (texte, discours interaction) pratiquées en France depuis les années 1970 reposent en effet sur une approche dualiste, décrivant le contexte comme extérieur au discours, comme le montrent les termes extralinguistique, extradiscursif, ou l'expression extérieurs du discours issue de la théorie du discours

(2) J'appelle logocentrées les approches de la langue et du discours qui posent les matières langagières comme objet central et prioritaire de l'analyse linguistique, au détriment des éléments environnementaux, autrement dit écologiques, qui selon moi entrent pleinement dans leur constitution.

(3) Je rappelle la célèbre formulation de Pêcheux et al. : « [...] le lien qui relie les "significations" d'un texte aux conditions socio-historiques de ce texte n'est nullement secondaire, mais constitutif des significations elles-mêmes » (Pêcheux et al. 1971, in Maldidier 1990 : 141). 
marxo-freudienne. De ce fait, on peut les considérer comme logocentrées, ce qui veut dire que les observables sont de nature «purement» langagière, ce qui signifie que les observables sont élaborés à partir du processus de production par les locuteurs, considérés comme des sujets séparables des environnements (c'est ce que Schaeffer appelle l'exception humaine dans son livre de 2007).

Les synthèses récentes sur la question du contexte présentent des approches qui ne remettent pas en cause fondamentalement le dualisme linguistique/extralinguistique : dans le numéro de Pratiques consacré à cette question (Achard-Bayle (éd.) 2006), un article de Moirand défend une conception dynamique du contexte, articulée sur une certaine forme de prise en compte de l'ensemble de l'environnement. Cette prise en compte s'arrête cependant aux « indices de contextualisation de niveaux divers au fur et à mesure de l'avancée du recueil des données et des analyses effectuées »; Moirand indique également que «le contexte socio-historique [...] entre de manière exhibée ou cachée dans les configuration discursives » (Moirand 2006 : 43). L'idée d'une « entrée » d'un élément dans un autre suppose deux réalités différentes : le contexte est donc bien extérieur aux productions langagières. Dans un récent article de synthèse sur le contexte, Kerbrat-Orecchioni dresse une typologie qui présente la même conservation du dualisme linguistique/extralinguistique : le rapport entre les deux est vu comme une influence, ou une modification, mais non comme une réelle constitution (Kerbrat-Orecchioni 2012).

\subsection{Une approche symétrique}

Pour éviter les risques d'une approche logocentrée, il faut déplacer l'objet de l'analyse et observer, non pas les énoncés seuls, mais l'ensemble du dispositif dans lequel ils sont produits, à la manière de E. Hutchins, qui, dans la perspective de la cognition distribuée, considère que, dans l'activité de pilotage d'un avion, c'est l'ensemble du cockpit, instruments de mesure compris, qui parle, et non seulement le pilote et le copilote (Hutchins 1994) : ce dispositif, je l'appelle environnement, appartenant à un dispositif théorique « symétrique».

J'appelle « linguistique symétrique » une approche des phénomènes langagiers postulant que les unités non linguistiques participent pleinement à l'élaboration de la production verbale, au sein d'un continuum entre verbal et non verbal, et non plus une opposition. Le terme symétrique st emprunté à «l'anthropologie symétrique » de B. Latour, pour qui on ne peut faire de la sociologie avec du social uniquement, car il faut tenir compte de la nature «assemblée » des phénomènes sociaux (Latour 1997). De même, je pense que l'analyse linguistique ne doit pas se contenter de porter sur des faits langagiers constitués uniquement de «langage» au sens traditionnel du terme, mais que la définition du langagier doit s'étendre à et s'enrichir de matérialités sociales, culturelles, environnementales, objectales, etc.

Une approche symétrique considère donc que les unités dites « extralinguistiques » participent pleinement à l'élaboration de la production des énoncés, au sein d'un continuum entre verbal et non verbal, et non plus une opposition (Paveau 2009). Ce point de vue n'est pas entièrement nouveau, puisque certains chercheurs en sciences du langage ont proposé des perspectives de ce type. Mais comme le soulignent Brassac et al. 2008a, cela reste une pétition de principe : « [...] although the ecology of action is becoming a key topic in various disciplines, studies that explicitly take artifacts and gestures into account — both theoretically and empirically — as they are actually mobilized by participants in interaction have remained underdeveloped » (2008a : 208). 
L'interactionnisme et la psychologie sociocognitive sont ce que l'on pourrait appeler des travaux « symétriques ». Les interactionnistes ont proposé dès les années 1980 d'intégrer les éléments de l'environnement dans l'analyse linguistique. Dans ses Éléments de pragmatique linguistique en 1981, Berrendonner propose par exemple de prendre en compte l'aspect signifiant de tous les éléments de la réalité, y compris les objets. Les chercheurs de l'école de Genève, autour de Bronckart et du courant de l'interactionnisme sociodiscursif, pratiquent aussi cette linguistique de l'extérieur, à partir des propositions de Vygotski et de l'école russe de la théorie de l'activité. Il faudrait aussi mentionner l'analyse conversationnelle issue de l'ethnométhodologie (Schegloff, Sacks, Mondada par exemple), qui développe des approches multimodales. Dans ces travaux cependant, si le sujet n'est plus logocentré, il reste cependant quelque peu " corpocentré » et le dualisme corps-monde est conservé. Dans le champ français, c'est plutôt du côté de la psychologie sociale et cognitive qu'il faut aller chercher des approches symétriques. Brassac, par exemple, plaide activement pour une analyse située et véritablement interdisciplinaire des phénomènes langagiers : dans son étude des interactions dans un hôpital autour de l'informatisation d'un service, il intègre pleinement le rôle des objets (Brassac et al. 2008a), comme dans son analyse de l'émergence de connaissances dans un processus collectif entre informaticiens et agronomes (Brassac et al.2008b). Plus récemment, le courant de la mediated discourse analysis prend comme unité d'analyse l'action, déplaçant lui aussi l'objet de l'analyse linguistique vers un dispositif complexe (Scollon \& de Saint-Georges 2003, Scollon \& Scollon 2003).

Quand les contextes ne possèdent pas prioritairement de matérialité concrète, mais une existence plutôt représentationnelle (ce qui peut être le cas du discours d'enseignement, bien que ses matérialités spatiales et objectales soient pourtant essentielles), ce type d'analyse peut être mené. Quand, en revanche, le contexte est constitué et même très largement défini par des réalités non langagières, ce qui est le cas des univers discursifs numériques, définis et même constitués par des paramètres technologiques, les propositions théoriques et méthodologiques doivent faire évoluer les épistémès et présupposés des disciplines texte-discours.

Il faudrait envisager de sortir du logocentrisme pour prendre en compte l'ensemble de l'écosystème des productions verbales, contenant l'ensemble des données qui nous entourent, qu'elles soient humaines ou non humaines. Ces données sont constitutives de nos productions langagières, et ne sont pas simplement un milieu qui leur serait extérieur. Dans cette perspective, les éléments dits inertes ou non doués de parole constituent quand même des agents psychiques qui participent de la production verbale et de la circulation des informations ${ }^{(4)}$. Artefacts, objets naturels, animés non humains, dispositifs techniques, configurations spatiales, etc., tous ces éléments doivent être pris en compte et intégrés au processus de production des discours. Un récent numéro de la revue Semen est entièrement consacré à cette question (Paveau (dir.) 2012b). Intitulé Texte, discours, interaction. Nouvelles épistémologies, il contient entre autres un article de de SaintGeorges sur la mediated discourse analysis et le détail de sa prise en compte des matérialités actionnelles (de Saint-Georges 2012), ainsi qu'une synthèse de ma part sur les rapports entre discours et réalités (Paveau 2012c).

(4) Sur cette question, voir Paveau 2007, 2009, 2012a, Paveau \& Rosier 2010. 


\section{Le genre de discours, du logocentré au composite}

Le genre de discours, entré en sciences du langage au début des années 1980, à partir de la pénétration en France des travaux de Bakhtine-Volochinov, est aussitôt devenu l'objet d'un débat définitionnel. Il s'agit en effet d'une notion plutôt large et puissante, qui offre donc une prise certaine à la discussion théorique.

\subsection{Des critères pour le genre}

«Même abondamment retravaillée depuis les années 1980, cette notion reste un des lits de Procuste de toute réflexion sur le discours ». Par cette réflexion, Maingueneau (2004 : 107) signale que le genre de discours oscille globalement entre une définition sociale (trop large) et une définition linguistique ou textuelle (trop étroite), aucune solution n'étant vraiment satisfaisante pour rendre compte d'une notion complexe dans l'analyse de laquelle entrent plusieurs paramètres.

Dans le classement qu'elle propose en 1999, Branca-Rosoff fait apparaître également ces deux grandes orientations, l'une plutôt sociale, et l'autre plutôt formelle, l'ensemble rassemblant des modèles et approches variées. À ces deux critères, social et linguistique-textuel, s'ajoute chez certains un critère cognitif, souvent articulé à l'un des deux premiers : Moirand parle de « représentation sociocognitive intériorisée » (2003), Rastier de dimension sémiotique et symbolique et du genre comme « lieu sémiotique de l'intersubjectivité» (2001a, 2001b) et Adam de "généricité comme nécessité socio-cognitive »(2005). La dimension cognitive permet une saisie de la notion de genre intégrant des paramètres représentationnels parfois implicites, voire tacites ${ }^{(5)}$ : on passe du genre dans le texte ou dans le social, au genre « dans la tête », si l'on peut dire.

Ces trois critères ne sont évidemment pas exclusifs les uns des autres et leur articulation permet de rendre compte de manière mixte d'une notion elle-même complexe et hétérogène. D'ailleurs, cette hétérogénéité $\mathrm{m}$ 'amène à préférer parler d'un « cadre générique » plus que d'un genre à proprement parler, comme une catégorie trop précise qui aurait ses étiquettes et ses exemplaires.

Les éléments définitionnels avancés par différents chercheurs, et que je considère comme acquis, éventuellement au prix d'une petite réinterprétation, sont les suivants :

— l'idée d'une stabilisation (ou d'une régulation, d'un programme ou encore d'un modèle) impliquant une certaine fixité ;

- l'idée d'une disponibilité cognitivo-sociale activable dans les productions verbales (les «formes du genre » selon Bakhtine);

- la conception du genre comme pratique empirique dans la réalité sociale, la praxis impliquant la prise en compte de la référence et de la réalité expérientielle.

Ma définition du genre de discours sera minimaliste : un ensemble de cadres collectifs pré-, extra- et intra-discursives, constitutifs de l'élaboration-interprétation des énoncés. Cette définition est élaborée dans le cadre de la théorie des pré-

(5) Je distingue le tacite, qui n'est pas destiné à être explicité, de l'implicite, qui peut être explicité. Le tacite est en quelque sorte un silence signifiant, alors que l'implicite est un silence signifiable. 
discours développée dans Paveau 2006. Je définis les énoncés comme des productions matérielles langagières en situation. Je ne parlerai pas de texte car mon approche n'est pas textualiste; comme je l'ai signalé plus haut, mon objet d'analyse est le discours comme énoncé dans son environnement, les deux étant co-constitutifs. Je m'écarte donc du choix d'Adam d'une « analyse du discours comme étude des textes empiriques ", impliquant que les genres de discours sont équivalents à des genres de textes $(2011 \mathrm{a}: 31)^{(6)}$. Je souhaite donc maintenir la notion de discours en ne séparant pas les manifestations intralinguistiques des contraintes extralinguistiques : je considère qu'il existe un continuum entre l'intradiscursif (la matière langagière) et l'extradiscursif (le contexte, ou environnement discursif) et non une distinction voire une opposition entre les deux. Ma perspective est intégrative, de type non dualiste ou post-dualiste.

\subsection{Le genre dans la technologie}

Cette perspective permet d'intégrer un élément essentiel, la matérialité technologique de la production des énoncés, négligée par la linguistique des discours et des textes, trop logocentrée, je l'ai dit plus haut, et même "égocéphalocentrée ", pour reprendre le terme que Brassac emprunte au sociologue Kaufmann ${ }^{(7)}$. L'ouvrage récent d'Adam sur les genres de récit (Adam 2011b) me semble confirmer ce logocentrisme, d'autant plus que le corpus est narratif.

J'ai proposé dans Les prédiscours en 2006, puis dans des publications ultérieures, la notion de « technologie discursive» (Paveau 2006, 2007, 2009, 2012a) pour rendre compte de la nature composite ou assemblée ${ }^{(8)}$ des productions langagières, contre le logocentrisme de la langue " en elle-même " issu du programme saussurien. Je ne suis évidemment pas la première à poser ce métissage entre le langage et autre chose que lui-même, qui fonde ma "linguistique symétrique », mais il ne me semble pas que les propositions antérieures aient été entendues à leur juste mesure : le mainstream des recherches linguistiques reste centré sur l'objet langue, et même les perspectives multimodales sont un peu marginales.

En ce qui concerne le genre, je pose son hétérogénéité comme l'un de ses traits principaux, particulièrement sur l'Internet : les genres de discours sont co-constitutifs des matérialités technologiques et des dispositifs communicationnels propres aux univers discursifs numériques. Les programmes génériques des discours numériques sont élaborés au sein de l'écosystème de l'Internet. Le genre de la « demande d'amitié » par exemple, issu de l'écosystème du réseau social Facebook, est par définition composite, techno- et -discursif. Cette demande passe

(6) Voir par exemple le très intéressant schéma de la page 34, intitulé « Niveaux ou paliers de l'analyse du discours ». C'est le texte qui est au centre du schéma comme l'objet de 1'analyse textuelle des discours, et non les discours.

(7) « Il est vrai qu'une grande partie des études conduites en psychologie sont largement ancrées dans un égocéphalocentrisme qui plombe, selon certains [Kaufmann 2001], leur capacité à appréhender la réelle nature des processus en jeu dans l'être-au-monde du sujet humain. Ainsi les solipsisme et mentalisme qui marquent par exemple la psychologie de la cognition laissent-ils en filigrane la question du rapport entre l'entité "pensante" et le monde "pensé"» (Brassac 2007 : 159; Kaufmann J.-C., 2001, Ego. Pour une sociologie de l'individu. Une autre vision de l'homme et de la construction du sujet, Paris, Nathan).

(8) Le terme d'assemblage est utilisé par Latour pour désigner la matérialité composite du «social» et par les chercheurs qui travaillent sur les continuums entre des matières qui ne sont pas de même nature, de type homme-machine par exemple. 
obligatoirement par un bouton (en français : « ajouter»), sur lequel il faut cliquer, en accompagnant (ou pas) ce clic d'un message scriptural. La demande d'amitié est donc un genre de discours constitutivement technodiscursif, dont il ne me semble guère possible de faire une analyse au sein d'une approche logocentrée.

Cette hétérogénéité du genre des discours en ligne est donc en partie due à la matérialité de la production des énoncés, qui justifie à mon sens une approche technologique ou technodiscursive de la notion de genre, pour rendre compte d'un certain nombre de formes récentes (et moins récentes d'ailleurs) dans lesquelles la contrainte technologique est nécessaire (au sens philosophique du terme) : le genre est aussi (et pour moi : surtout) dans les artefacts, conçus comme des réalités augmentées, au bout des doigts et des claviers, et sur nos écrans et tablettes.

Je définis le genre de discours composite comme un genre au sein duquel les énoncés n'ont pas une nature «purement» langagière, mais en partie constituée de matériau technologique : écran, timeline, avatars, liens, boutons, bookmarklets, etc.

Pour récapituler mon approche des discours natifs en ligne, je souhaite montrer comment ce que j'appelle la technologie discursive est constitutive des productions verbales sur la toile, constitutive au sens d'impliqué dans la nature même des énoncés, et non considérée comme un simple « outil » de production verbale.

\section{Le web 2.0, un écosystème technolangagier}

Avant de présenter le réseau de microblogging Twitter, je précise quelle est ma conception des univers numériques, quels sont mes observables et le mode de présentation des éléments du corpus.

Les mondes de l'Internet, plus de vingt ans après leur intégration aux usages quotidiens, et au moment où le web 2.0 semble laisser progressivement la place à l'Internet des données, font encore l'objet d'un débat assez vif, comme le montre le succès de l'ouvrage de Carr, The Shallows : What the Internet Is Doing to Our Brains, traduit en français par Internet rend-il bête? (Carr 2011 [2010]), après celui de son célèbre article "Is Google Making Us Stupid? » (The Atlantic, 2008). Pour aller vite, le débat oppose une conception de l'Internet comme lieu social à part entière (services, relations, information, écriture, networking, militantisme, loisirs, etc.) à l'Internet comme espace de risque pour la société (cyberharcèlement, prédation sexuelle, information incontrôlée, rumeurs, développement des extrémismes, fraudes, etc.) ; une image de l'Internet comme espace d'augmentation de nos capacités (écriture augmentée, développement de l'intelligence collective, multiplication des identités numériques, accroissement et partage des savoirs, etc.), à l'Internet comme lieu de dégradation de ces capacités (appauvrissement des gestes de lecture et d'écriture, superficialité de la pensée, facticité des relations, etc.). Mais surtout, l'opposition entre virtuel et réel, qui a pourtant largement été remise en cause par une vingtaine d'années de travaux et d'usages, reste tenace, et fait peser sur les univers numériques le soupçon de leur inauthenticité, voire de leur inexistence.

Je considère que l'Internet fait partie de notre monde, qu'il est réel comme le sont les interactions, les communications et les créations qui s'y élaborent. J'écarte donc le terme virtuel, que je conserve spécifiquement pour les jeux en ligne qui élabore des mondes virtuels (par exemple World of Warcraft) ou les simula- 
teurs de monde (par exemple Second Life). Pour distinguer les ordres de réalités, j'emploie les termes en ligne vs hors ligne, et parfois IRL (In Real Life) parce que le terme est lexicalisé et très utilisé sur l'Internet, en particulier dans les réseaux sociaux. Je pense que les deux faces positive et négative de cet univers coexistent, comme dans la vie hors ligne, et que les chances et les risques n'y sont pas plus importants, mais prennent des formes différentes.

J'essaie également de m'écarter de la problématique ancien $v s$ nouveau, au sens où il faudrait inscrire les écrits numériques dans des genres préexistants. Pour Marcoccia 2005, par exemple, « le courrier électronique est une forme (numérique) de billet », le billet étant un message bref dans son écriture, son contenu et sa forme, apparu au $17^{\mathrm{e}}$ siècle lorsque les épistoliers ont éprouvé le besoin de se dispenser des formules de politesse conventionnelles. Les énoncés sur Twitter sont régulièrement comparés à des formes brèves anciennes : 1'épigramme, le haïku, l'aphorisme. Ces comparaisons posent deux problèmes : d'abord, elles ne permettent pas d'observer une forme de manière écologique, c'est-à-dire dans son environnement cognitif, social, culturel propre ; ensuite elles sont en général argumentatives, dans la mesure où elles servent à défendre ou à légitimer des formes condamnées, dans une perspective normative. Ces deux points me semble ascientifiques; il est plus intéressant de voir dans les écrits numériques, comme dans les univers numériques en général, des activités contemporaines, à la fois inscrites dans des traditions et ouvertes à des transformations.

Mes observations portent sur les discours numériques natifs, c'est-à-dire produits dans 1'écosystème numérique du web 2.0 : réseaux sociaux (Twitter, Facebook, Diaspora, Researchgate, Academia, Pinterest...), blogs, sites, wikis, plateformes de curation (Scoopit, Pearltrees), outils de veille (Google Reader, Diigo, Netvibes), outils d'annotation en ligne (Annotate, Lino it, MyStickies). Ces discours sont fondamentalement écrits, même s'ils peuvent être métissés de documents sonores ou hypermédiatiques, ou s'ils peuvent être oralisés au moyen de certaines applications. Le terme numérique recouvre en effet, en ce qui concerne les productions écrites, des réalités sémiotiques différentes :

- des discours imprimés numérisés pour l'analyse linguistique par exemple, donc non natifs mais qui acquièrent des traits natifs, par exemple la navigabilité ; constituant des bases de données, ils sont mis en ligne ou pas ; c’est le cas des corpus annotés des talistes par exemple;

- des discours imprimés numérisés par scannage ou mise en ligne, dans un contexte éditorial, avec les mêmes propriétés acquises ; ils sont en ligne ; c'est par exemple le cas des revues numérisées sur le portail Persée ;

— des discours numériques natifs, produits directement en ligne, qui peuvent conserver la linéarité scripturale des écrits imprimés (pas de liens hypertexte, pas de réticularité, comme c'est souvent le cas sur les blogs littéraires par exemple) ou prendre des formes réticulaires grâce à l'hypertextualité (systématique et même automatique dans le cas des réseaux sociaux) ${ }^{(9)}$.

Le mode de présentation des corpus dans un article doit donc être à mon sens celui du travail sur l'écran en ligne, ce qui n'est évidemment possible qu'à l'oral, dans le cadre d'un colloque ou d'un séminaire. Cela pose évidemment un pro-

(9) Sur cette question voir par exemple le carnet de recherche Technologies discursives, http://technodiscours.hypotheses.org/ 
blème éditorial si les publications des chercheurs qui travaillent sur l'Internet sont seulement imprimées, sans version numérique en ligne. Les chercheurs qui ont abordé les langages et discours de l'Internet ${ }^{(10)}$ ont d'ailleurs le plus souvent présenté des corpus discursifs extraits de leur environnement, mettant de côté la matérialité de l'environnement technodiscursif de l'Internet. Mais il y a là une perte scientifique importante, les observables étant alors considérablement réduits. Il est toujours possible de publier des captures d'écran (même en noir et blanc si le procédé est trop coûteux), qui me semblent le minimum requis pour assurer une bonne lecture des données présentées (c'est le procédé adopté dans le présent article).

\section{Matérialité des technologies discursives : l'exemple de Twitter}

\section{Illustration 1 : le compte de François Hollande}

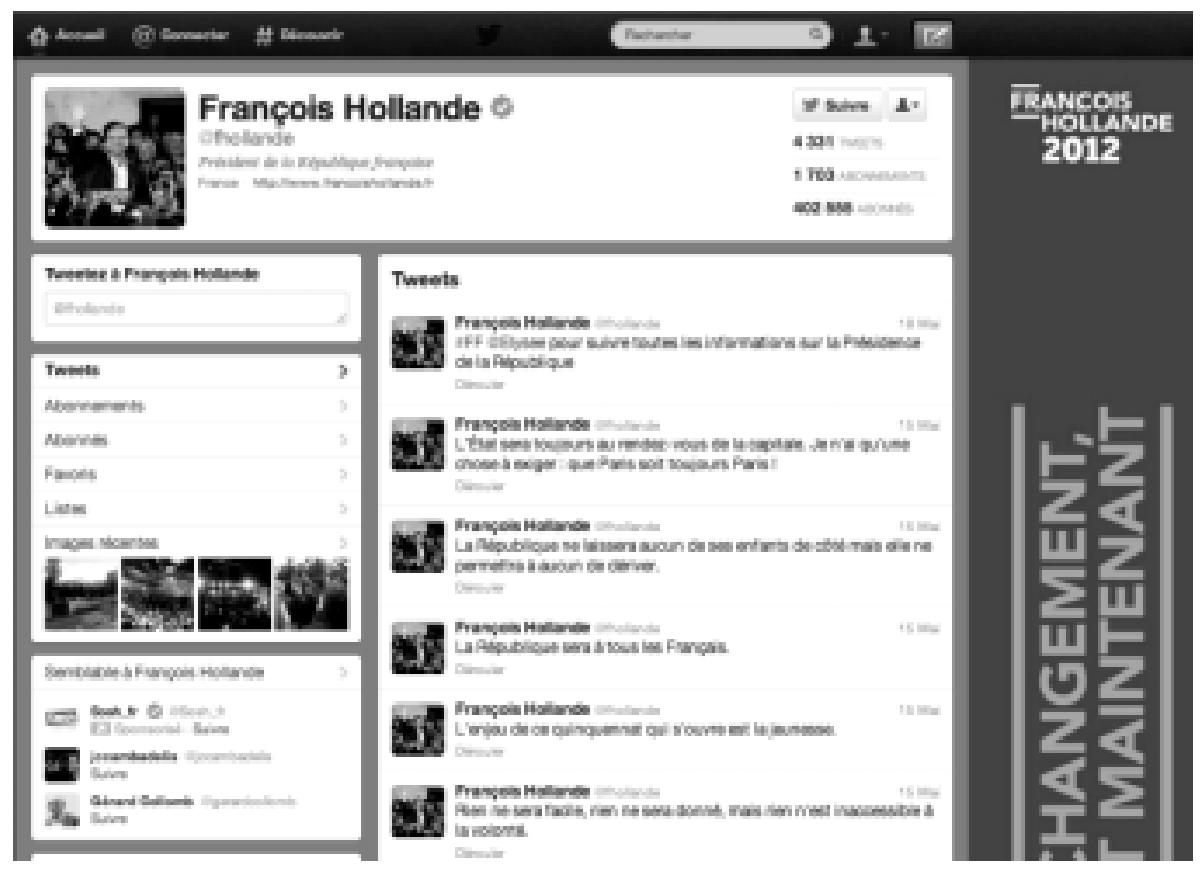

(10) Je pense particulièrement au courant de la CMO (Communication médiée ou médiatisée par ordinateur ), qui pose une médiation et non une intégration, et concerne plutôt des productions antérieures au web 2.0 et des productions téléphoniques (SMS). Avec la CMO, on est vraiment sur l'ordinateur comme outil externe et non pas comme prolongement ou augmentation du scripteur (par exemple : Marcoccia, Panckhurst). Ces travaux sont prolongés de manières diverses : des travaux sont actuellement menés en ELAO (Enseignement des Langues Assisté par Ordinateur), par Desmet \& Rivens Mompean par exemple. Le terme assisté, maintenu dans l'appellation, signale toujours cette extériorité de l'outil informatique. Il existe également des travaux sur les « discours sur l'Internet » en analyse du discours et sociolinguistique, essentiellement autour de la question de l'écrit (à partir des travaux pionniers de Jacques Anis), des formes morphographiques, de l'écriture sms, des abréviations, smileys, etc. Un axe de travail s'est développé autour de la question à mon avis discutable de l'oralité dans les écrits en ligne (sur les forums essentiellement). Les travaux sur les réseaux sociaux commencent à émerger. 
Le réseau de microblogging Twitter a été lancé en 2006, deux ans après le réseau social Facebook. Au départ, il devait permettre aux utilisateurs de décrire instantanément et brièvement ce qu'ils étaient en train de faire. Le slogan d'origine était en effet : «What are you doing ? » ${ }^{(11)}$. Le service est d'abord utilisé par l'intermédiaire des SMS (Short message service) sur les téléphones, qui n'autorisent que 160 caractères (blancs et ponctuation compris). Twitter en « prélève » 20 pour le nom d'utilisateur précédé de 1'@ (le pseudo) et c'est ainsi que les fameux 140 caractères apparaissent. La contrainte est donc technologique au départ, puis elle est devenu une routine (il est techniquement possible d'augmenter le nombre de caractères sur Twitter et il existe par ailleurs une application pour des tweets longs (longtweet.com), qui transforme un tweet long en image republiée ensuite sur Tweeter.

Il y aurait en France, au 31 janvier 2012, 5,2 millions de comptes Twitter, mais la moitié d'entre eux peut-être sont actifs. Le réseau présente une utilisation simple et conviviale : à l'ouverture du compte, on choisit un pseudo précédé de 1'@(@mapav8 pour moi), court de préférence de manière à ne pas occuper trop de place dans les réponses et les retweets, un mot de passe, un avatar; on écrit sa biographie en 160 caractères (voir les exemples ci-dessous, qui utilisent diversement les possibilités technoscripturales de cet espace), on choisit des applications pour l'alimentation automatique par ses blogs par exemple (les billets sont signalés automatiquement sur Twitter), ou pour signaler sa position géographique (réseau Foursquare).

\section{Illustration 2 : les «bios » et avatars de Twitter}
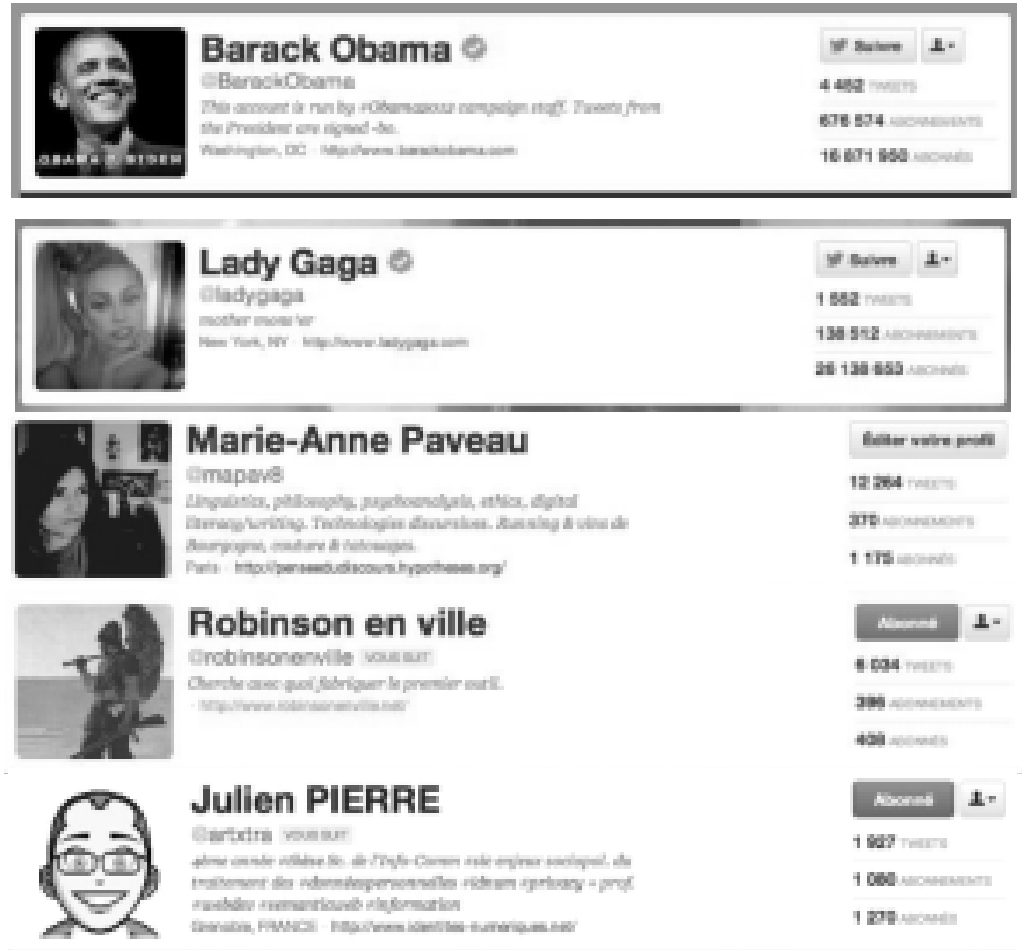

Julien PIERRE

cintatrin voum

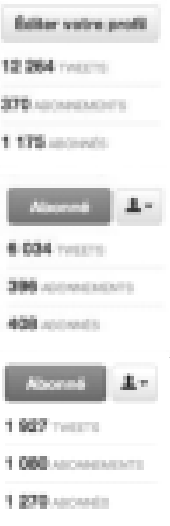

(11) Il est devenu « What's happening? » et désormais « Compose new tweet» («Écrire un nouveau tweet » en français). 
Une fois inscrit on s'abonne (on follow et on devient alors un follower) et se désabonne (unfollow) librement à des comptes qui sont la plupart du temps ouverts (les comptes privés accessibles sur demande sont possibles mais ne correspondent pas à la tradition de Twitter qui est plutôt libriste et favorable à l'open access), on peut bloquer un abonné ou le signaler comme spam, on peut aussi faire des listes de followers si l'on veut trier ses informations. Twitter est asymétrique (contrairement à Facebook) : on peut suivre quelqu'un sans le lui avoir demandé, sans qu'il ne nous suive et réciproquement.

Il existe de nombreuses applications pour Twitter sans passer par le site officiel, qui possèdent des fonctionnalités supplémentaires et améliorent donc l'ergonomie du service, permettant de tweeter à partir d'un smartphone ou d'une tablette.

Une fois le compte installé, le/la «twittos/twitta», ou «twitteur/twitteuse » selon l'édition 2013 du Petit Robert ${ }^{(12)}$, peut se livrer à différentes activités technodiscursives sur son fil d'actualité ou timeline (mot abrégé en TL, et couramment utilisé au féminin : « une TL », « ma TL »).

\subsection{Des activités technoscripturales}

Twitter propose des activités technoscripturales en nombre restreint, parfois codifiées par des sigles à l'intérieur de la fenêtre des 140 caractères :

a. Tweeter un message (un tweet), en une ou plusieurs fois (on numérote alors les tweets).

b. Répondre à un tweet, dans le cadre d'un dialogue ou d'un polylogue, la réponse étant marquée par le pseudo de l'interlocuteur eu début du tweet.

c. Retweeter un tweet, activité symbolisée par le signe RT, ou MT (modified tweet) s'il est modifié, avec ou sans commentaire personnel marqué par / ou // ou $<$ ou une autre convention.

d. Livetweeter un événement, comme un colloque, une émission de télévision, un événement politique (la campagne présidentielle a créé une sorte de livetweet au long cours, marqué par les hashtags respectifs des candidats, comme \#FH2012 par exemple) ou une rencontre sportive. Le livetweet suppose 1'adoption d'un hashtag (ou mot-clic ou balise pour les Québécois), signalé par le symbole \#, qui rend le mot clicable, en permettant alors d'accéder de manière hypertextuelle à l'ensemble des tweets contenant le hashtag en question. Des outils en ligne ${ }^{(13)}$ permettent de rassembler tous les tweets concernés en un document (généralement un fichier pdf) : c'est la redocumentation, activité technodiscursive et informationnelle qui fait évoluer le web 2.0 vers le 3.0, le web des données.

e. Envoyer un DM (direct message), message privé, à ses followers.

(12) Sur le réseau, twittos et twitta sont majoritaires et twitteur et twitteuse pour le moment assez rares (selon mon impression d'utilisatrice et sans données quantitatives). Mon objectif n'est pas de mener ici une étude lexicographique du vocabulaire de Twitter. Je signale seulement que de nombreux néologismes, formés par «préfixation » des éléments $t w i t t$ - ou $t w$-, sont produits, dont certains se lexicalisent, comme : twittclash, twunch, twittapéro, twami, twelcome, twittécriture, twittérature. Les graphies sont instables : Twitter pour le nom du réseau, twitter et retwitter pour le verbe, tweet et retweet pour le nom du message.

(13) Par exemple Tweetdoc ou Tweet Archivist. 
Illustration 3 : les activités technoscripturales sur Twitter

a. Tweeter et b. Répondre

Marie-Anne Paveau comapmes

les amis, comment traduiriez-vous en anglais technodiscours,

technolangagier, technomot? que jemploie pr décrire le langage

natif en ligne

Derouler + Absondre At Supprimer Favori

Rose Amer ahose Amer

Bmapav8 technospeech technolinguistic technoword?

Dirouler

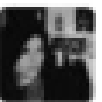

Marie-Anne Paveau clmmome

CRose Amer merdi bcp je note - je dois vérifier que ça ne fait pas ambiguite avec le sens de "langage" ou "mot DE LA technique", tu vois

- Masouer la conversation + Alipondro A Supprimer * Favon

$11: 54$ - 24 Juin 12 via wob - Detala

c. Retweeter

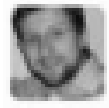

Jullen Hering PhD ejulen hering

FT Egayaneadourian Qques min de Casse Noisettes par Brigitte

Engerer parce que cette immense pianiste est partie hier goo.gl/1KhDU

Q Voir la vidbe + Alponde ta Aletweoter Faver

d. Livetweeter

Gayané Adourian cGayaneldourian

II parle ensuite des média sociaux,au coeur de l'action

aujourd'hui.Twitter permet d'identifier les influenceurs de l'espace urbain rexpoviz

Diroulor

Gayane Adourian bGayaneldouran

J'en étais suure II II parle maintenant de la vidéo que je viens de twitter $\mathrm{lo} / \mathrm{Hexpoviz}$

Dienculier

Gayane Adourian EGaryaneAdourian

Ayman montre le croisement qu'lis ont fait entre les données de trafic et de temperature a Saragosse \#expoviz

Derouive

e. Envoyer un " direct

message » en utilisant

une fenêtre dédiée

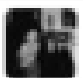

Marie-Anne Paveau

Voi ma page de profl

Messages prives
Listes
Aide
Raccourcis clavier
Parametres
Deconnexion




\subsection{Des formes technolangagières}

Le tweet exploite quatre catégories de formes langagières :

- des formes linéaires sans caractéristiques technolangagières autres que l'inscription sur support informatique ;

- des technomots comme le hashtag (précédé du croisillon \#) qui permet l'organisation de l'information par la mise en réseau de plusieurs messages et le pseudo (précédé de @) qui renvoie au compte du twitteur ;

— des liens (URL) qui permettent d'accéder à des sites ${ }^{(14)}$

- des émoticones ${ }^{(15)}$.

Ces formes peuvent se combiner de manière plus ou moins complexe : certains twitteurs ont une écriture purement linéaire (cas de la twittérature décrite plus bas), d'autres combinent les mots, les technomots, les liens et les émoticones de manière très complexe, comme le montrent les exemples suivants, qui nécessitent une bonne expertise des lois du genre :

Illustration 4 : des tweets particulièrement composites (29.06.2012)

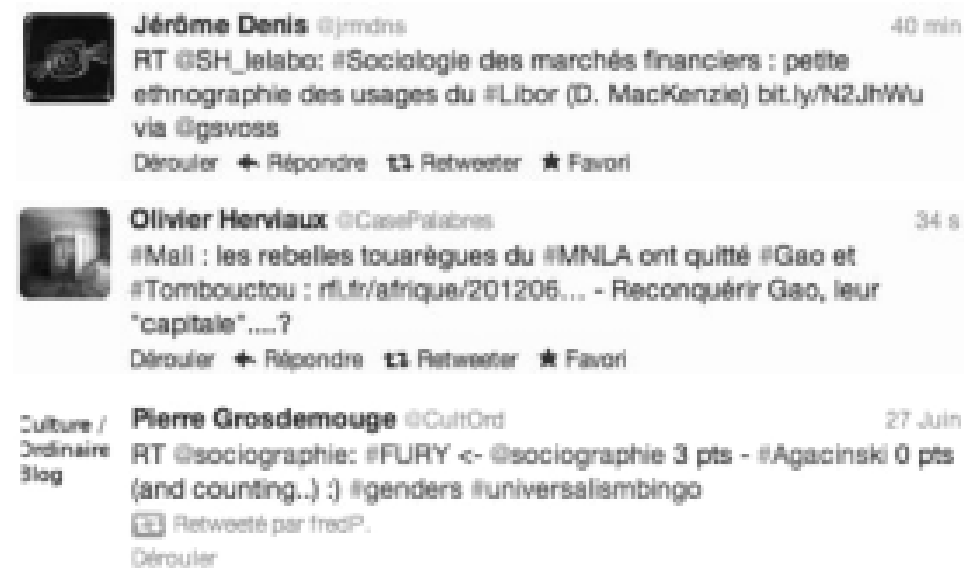

\subsection{Des pratiques technoculturelles}

J'appelle pratiques technoculturelles un ensemble de pratiques nées au sein de la communauté des twittos, et qui n'étaient pas inscrites dans le programme de départ du service en 2006. Ce sont en effet les usagers qui ont inventé par exemple le " followfriday », siglé en \#FF et doté du croisillon : tous les vendredi, les usagers peuvent indiquer à leurs abonnés des comptes à suivre en faisant précéder ou suivre leur message de ce hashtag. De même, le jeudi est traditionnellement le jour de la \#jeudiconfession, où il est de coutume de formuler un tweet qui soit ou ressemble à ou prétend être une confession, sur le mode ludique ou sérieux. À l'origine, le service Twitter n'a fourni que les possibilités techniques, que le techno- de tech-

(14) Il existe des raccourcisseurs d'URL qui permettent d'économiser des signes dans les tweets, ce sont des services auxquels il faut de s'abonner, et qui constituent des vrais dispositifs technolangagiers : la taille de la matière langagière est adaptée aux contraintes technologiques du réseau.

(15) On trouve également, mais plus rarement et surtout chez les twittos programmeurs (qui sont nombreux sur Twitter), des formes issues de l'Art ASCII. 
nodiscours ; ce sont les usagers qui, s'appropriant l'espace de communication, les formes d'écriture et la technologie, ont contribué à créer ce que j'appelle des formes technolangagières. On a donc là quelque chose qui ressemble à une communauté discursive, si on la définit comme un ensemble de locuteurs qui partagent des usages (et non des formes) et des rapports au langage et au discours.

De ces pratiques technoculturelles ressortissent également un nombre important de métadiscours procéduraux concernant l'utilisation du réseau : il existe de très nombreux tutoriels en ligne, produits par le service Twitter ou proposés sur différents sites et blogs, qui contiennent des discours d'apprentissage. C'est une originalité des réseaux sociaux sur la toile, qui s'accompagnent généralement de leur guide d'usage ou de « savoir vivre », contrairement aux réseaux sociaux IRL, pour lesquels la maîtrise des usages est plutôt du ressort du capital culturel et social $^{(16)}$. De fait il existe donc une sorte de «twittétiquette» sur le modèle de la netiquette, qui recommande un certain nombre de comportements technodiscursifs sur le réseau (par exemple : saluer, répondre aux tweets adressés, partager des contenus) et qui en déconseille d'autres (par exemple le « flooding », c'est-à-dire la diffusion, souvent automatique car générée par un lecteur de flux, de plusieurs dizaines de tweets à la suite).

Ces métadiscours peuvent constituer un indice d'une généricité nouvelle, soulignant qu'il existe un savoir faire scripturo-technodiscursif sur les réseaux, désormais stabilisé, partageable, transmissible et reproductible qui empêchent certains usagers d'intégrer le réseau Twitter : une réalité scripturale, culturelle, sociale et technique qui possède un air de famille, au sens sémantique du terme, avec la notion de genre.

\section{La généricité composite de la twittécriture : vers des technogenres}

Si Twitter constitue une communauté discursive et si les discours qui y sont produits sont moulés dans les formes d'un genre global, le réseau produit également des programmes génériques particulier, ou modifie des programmes existants. J'en examinerai deux, les genres pédagogiques-didactiques et les genres littéraires.

\subsection{Les (techno)genres de l'enseignement}

Twitter a été investi à partir de 2009-2010 par les enseignants du secondaire, de la maternelle au lycée, en particulier, dans l'espace francophone, au Québec, puis un peu plus tardivement en France. Une communauté enseignante passionnée de pédagogie et de numérique, parfois d'informatique et de technologie ${ }^{(17)}$, y propose des activités pour la classe, y invente des pratiques et y organise des conversations pédagogiques.

(16) C'est vrai pour l'époque contemporaine. Il y a eu longtemps des guides de savoir-vivre et de savoir-parler. Il est possible d'ailleurs que la tradition écrite du guide de savoir-vivre soit en quelque sorte remplacée, ou au moins continuée, par ces métadiscours en ligne.

(17) Je dis parfois car je fais une distinction nette entre informatique et numérique : comme d'autres, je pense que le numérique ne nécessite pas forcément de connaissances informatiques et que la programmation n'est pas une compétence nécessaire aux littéracies numériques. Mais cette question est fortement débattue dans les milieux éducatifs où se pose la question de l'enseignement de la programmation aux élèves. 
Au 26 juin 2012, il y a 172 twittclasses francophones, c'est-à-dire des classes qui ont mené au moins une activité pédagogique via Twitter. Elles sont régulièrement recensées sur le site «Twittclasses francophones ». Ces classes ont des comptes Twitter, ouverts officiellement et légalement dans le cadre de l'établissement. Les enseignants les utilisent de manières très diverses pour y organiser des activités pédagogiques en classe et hors de la classe. Toutes les activités ont bien sûr leur hashtag ou balise identificatrice. Voici quelques exemples :

_\#devoirpt. Annie Côté (@AnnieSentiers), enseignante de français au Québec, a été l'une des premières à organiser des exercices sur Twitter, comme le rapporte le Bulletin électronique du Conseil supérieur de la langue française :

Au printemps 2011, Annie Côté, une enseignante de français en cinquième secondaire de l'école secondaire Saint-Pierre et des Sentiers, de l'arrondissement Charlesbourg de la ville de Québec, a proposé à ses élèves de rédiger huit devoirs sur la plateforme Twitter, à raison d'un devoir par semaine. Ces devoirs devaient, comme l'impose le site de microblogage, comporter au maximum 140 caractères, incluant la ponctuation et les espaces. Chaque devoir devait également respecter un thème précis, lequel différait chaque semaine (http://www.cslf.gouv.qc.ca/no-cache/infolettre-du-francoscope/article/twitter-et-lenseignement-du-francais-ausecondairenbsp-une-entrevue-exclusive-avec-annie-cot-1/)

—\#12hommes. Au Québec également, Jean Doré (@jeandore), dépité de voir sa classe de $5^{\mathrm{e}}$ secondaire sans réaction devant le film Douze hommes en colère de Sydney Lumet, projeté dans le cadre d'un travail argumentatif sur la peine de mort, organise des activités sur Twitter, avec la balise \#12hommes (la description de l'activité figure sur un wiki consacré aux activités numériques au collège : http://dorej.wikispaces.com/message/view/home/36229164).

— \#twittcorrigé. Delphine Regnard (@drmlj), professeure de Lettres classiques en France a ainsi diffusé le corrigé d'un devoir de ses BTS du lycée SaintExupéry.

— \#tweetconte. Nathalie Couzon (@nathcouz), organise quant à elle en décembre 2011 une cueillette de "mots des contes »sur Twitter pendant une semaine, mots qui sont ensuite fournis aux élèves qui rédigent des contes en dix tweets (l'ensemble des mots est recueilli ici : http://randonnee.effetdesurprise.qc.ca/ $? \mathrm{p}=1610)$.

— \#conseilsorcière. Alexandre Acou (@Alex_Acou), professeur des écoles dans la région parisienne, a fait rédiger à ses élèves de CM1 des énoncés brefs sur Twitter, destinés à entrer en dialogue avec ceux d'une twittclasse anglaise. L'activité est présentée et racontée dans un dossier Storify par Stéphanie de Vanssay (@2vanssay) :

À la manière de Jean Tardieu écrire des conseils de sorcière en employant l'impératif, puis les tweeter avec la balise \#conseilsorciere. Ensuite les collégiens de @IDEM_in_English ont relevé le défi d'en rédiger à leur tour en anglais avec la balise \#witchadvice (http://storify.com/2vanssay/les-conseils-sorcieres-des-elevesde-classe-acou)

Il existe un outil (Tweetdoc) qui permet de rassembler tous les tweets comportant telle ou telle balise et de les enregistrer dans un fichier pdf (pour \#conseilsorcière, voir annexe 1). Ce document propose une (re)textualisation d'un ensemble de tweets épars, textualisation assurée par la redocumentation technique, et par la 
lecture : un programme générique qui nous mène vers un technogenre. En effet, si la teneur des exercices en soi n'est pas nouvelle, leur mise en œuvre sur le réseau social les reprogramme en quelque sorte, et c'est à de nouveaux genres pédagogiques que nous avons affaire, parce qu'ils constituent des assemblages composites entre le langagier-discursif et le technologique.

Mais Twitter est également un lieu informel d'échange et de formation pédagogique : chaque mercredi pendant l'année scolaire, les twitteurs enseignants francophones de tous pays peuvent échanger sur un thème pédagogique donné, au sein du \#claved (pour « clavardage éducatif», voir annexe 2). Cette activité de formation et d'information, proposée par des Québécois, se déroule à heure fixe (midi heure québécoise), et produit des échanges et des savoirs pédagogiques et didactiques. C'est un projet pensé et encadré, qui s'inscrit dans un objectif de formation continue, de dialogue collaboratif et de création de liens entre des enseignants parfois très isolés (voir le "plan de relance» du Claved sur le site dédié http://claved.wordpress.com/). Il s'agit là d'une programmation générique qui me semble assez nouvelle, même si l'on peut y voir un genre de discours proche de celui qui est produit dans les « groupe de pratiques » qui existent IRL. Dans le cas du \#claved, les lois du genre sont constitutivement assemblées avec le dispositif technologique : brièveté obligatoire des interventions, technodiscursivité, pseudonymat éventuel des participants, polylogalité écrite, conversation augmentée (par la diffusion de liens par exemple), coprésence distante à travers les continents.

Dans une autre perspective, plus documentaire, Géraldine Duboz (@gduboz) produit une « revue des tweets » mensuelle, concernant 1'histoire, la géographie, l'éducation civique, la pédagogie sur son site Revue de tweets, qui héberge égalent une revue en français et lettres classiques alimentée par Delphine Regnard (http://revuedetweets.wordpress.com/).

L'enseignement trouve donc dans le réseau Twitter un autre espace discursif et d'autres modalités d'enseignement-apprentissage.

De la technodiscursivité propre au dispositif informatique, semblent donc naître des technogenres dont la description linguistique reste à faire.

\subsection{Le paradoxe de la twittécriture linéaire : la twittérature}

Sur Twitter, on fait de la twittérature. Sur le site de l'Institut de Twittérature Comparée (http://www.twittexte.com), on trouve cette présentation :

L'Institut de Twittérature Comparée (ITC) existe parce que Twitter existe. Parce que la littérature existe. Parce qu'il est possible de rédiger des textes en moins de 140 caractères (espaces compris). Parce qu'on peut être drôle et intelligent à la fois et vice versa. Parce qu'on peut être aussi simultanément dense et léger, brut et subtil, lent et rapide, cérébral et viscéral, poétique et discursif. La twittérature existe enfin parce qu'une nanorhétorique des antithèses, oxymorons compris, stimule l'essor et l'éclat; elle y trouve sa piste d'envol et son champ d'exploration (page «L'ITC).

L'ITC propose un manifeste qui contient les deux paragraphes suivants :

La twittérature est à la rature, ce que le gazouillis est au chant du coq. Les uns vantent l'alexandrin, d'autres jouent du marteau-piqueur.

La twittérature est la somme de récits, aphorismes et autres apophtegmes. Une cacophonie de gazouillis que symphonise harmonieusement l'ITC (page « Manifeste). 
Ces quelques éléments donnent une première description de la twittérature, née avec le réseau Twitter, et à laquelle les twittérateurs attribuent un précurseur : Keitai Shosetsu, qui, au Japon, dans les années 2006-2007, produit les premiers romans écrits sur téléphones cellulaires, appelés " celluromans » ou «cellu-lit». Toujours selon les informations de l'ITC, la twittérature se réclame des haïkus japonais, des romans-feuilletons du XIXe siècle, des cadavres exquis des surréalistes et des expériences littéraires de 1'OuLiPo. En 2008 Laurent Zavack rédige une nouvelle érotique sur Twitter, appelée érotwitt, On l'appelait Sodomy, « fiction écrite uniquement avec des SMS que s'envoient les personnages de l'histoire. Il serait le premier au monde à avoir publié sur papier, en mai 2009, un "twitteroman", un roman entièrement pré-publié sur son compte Twitter» (ITC, page « Origines »). La même année, Thierry Crouzet écrit un twiller (ou techno-thriller), roman interactif intitulé Croisade, constitué d'environ 5200 tweets postés entre le 25 décembre 2008 et le $1^{\text {er }}$ avril 2010 (les premiers sont disponibles sur le compte de1'auteur@tcrouzet, mais le roman a été édité sur support traditionnel).

Il semble que ce soit en 2009 qu' apparaît le terme twitterature, au moment de la publication de l'ouvrage d'Alexander Aciman et Emmett Rensin, Twitterature : The World's Greatest Books Retold Through Twitter (Penguin Books, traduit en français en 2010). Il s'agit d'une série de pastiches de plusieurs œuvres canoniques de la littérature et 20 tweets ou moins.

On trouve sur le site de l'ITC un annuaire raisonné des twittérateurs, dont certains sont devenus célèbres dans l'univers de la twittécriture et de l'écriture numérique en particulier : centquarante, twittlit et nanonouvelles font des récits ; pierrepaulpleau, nanopoesie ou fanfiole font de la poésie, machinaecrire et aurise font des textes dits « hybrides ».

Sur le plan technodiscursif, la twittérature est non technologique et c'est là que réside le paradoxe. Alors que la twittécriture est une techno-écriture par définition (usage des hashtags et des pseudos comme technomots clicables, liens hypertextuels, etc.), la twittérature est linéaire, c'est-à-dire non technique et non réticulaire :

En règle générale, elle ne s'affuble pas d'un fil corrompu par la discussion bilatérale, par le retweet (RT), par la citation (@), le hashtag (\#) ou le renvoi (http://www). Bien que Twitter soit un outil exemplaire pour acheminer des données brèves aux abonnés de son compte, le twittérateur ne retweete que très rarement. S'il le fait, souvent il s'amusera à contourner la règle de Twitter en faisant en sorte que son gazouillis soit contenu dans 140 caractères pile. Autrement, il n'écrit que des mots, il ne fabrique que des phrases.

Idéalement le fil de discussion du twittérateur est pur, il est monochrome. On n'y retrouve que les 26 lettres de l'alphabet et les signes de ponctuation. Des chiffres à la rigueur (surtout cette allusion au nombre cent quarante qui permet tant de clins d'œil et d'autodérision) peuvent parfois émailler la séquence ; mais aux chiffres, on préférera les lettres. Tant mieux disent certains si la pensée proposée tient dans 140 caractères, mais ce n'est pas forcément obligatoire (site de l'ITC).

Et effectivement, sur les exemples ci-dessous, la linéarité non technologique se voit à l'œil nu, par le simple jeu des couleurs (le tweet sans date : du 29.06.2012) : 


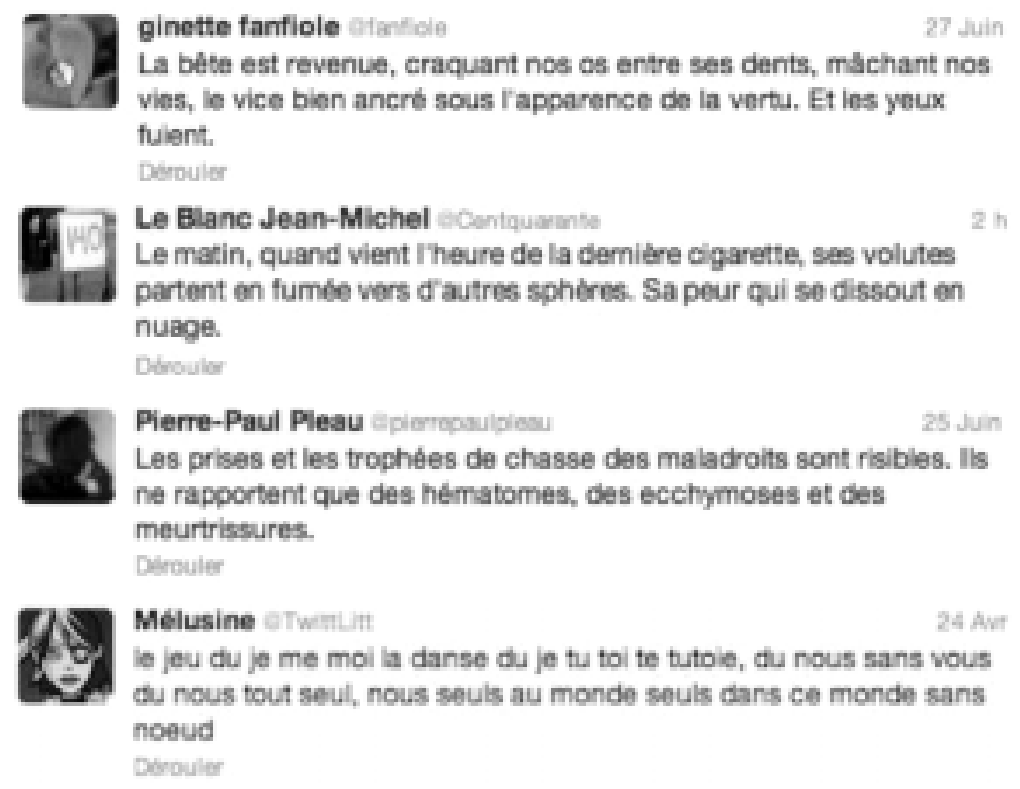

La twittérature, un technogenre ? Oui et non. Structurellement technique puisque métissant le logos pur de technologie numérique et informatique, elle refuse cependant que cette technologie affecte le logos. On se trouve donc devant la contradiction d'une techno-écriture logocentrée; aucune des marques délinéarisée de la twittécriture (@, \#, RT, liens, etc.)n'étant adoptée.

\section{Conclusion}

L'écriture numérique native possède des traits particuliers qui lui sont donnés par le dispositif technologique. Mais ces traits ne sont pas des traits « en plus », qui laisseraient le logos et le logocentrisme intacts. Ces traits affectent la nature même du langage, qui se métisse de technologie. Il nous faut désormais reconnaître qu'il n'y a pas que du langage dans le langage. De mon point de vue, la chose n'est pas nouvelle, mais les univers discursifs numériques la rendent explicite.

Le monde du réseau de microblogging Twitter produit un (techno)genre de discours qui est le tweet, produisant lui-même d'autres technogenres et technopratiques discursives qui sont en train de se stabiliser et de devenir reproductibles. Ces technogenres sont des aménagements de genres préexistants (en twittérature en particulier) ou des inventions de l'écosystème numérique, comme le \#claved. Il semble donc qu'il y existe des genres endémiques sur Twitter. Reste à savoir si les espèces seront transplantées dans d'autres environnements. 


\section{Bibliographie}

ACIMAN, A., RENSIn, E. (2009) : Twitterature : The World's Greatest Books Retold Through Twitter, London, Penguin Books [trad. française : 2010, La twittérature. Les chefs-d'œuvre de la littérature revus par la Génération Twitter, Paris, Éditions Saint-Simon].

ADAM, J.-M. (2005 [1999]) : La linguistique textuelle. Introduction à l'analyse textuelle des discours, Paris, A. Colin.

- (2011a [1992]) : Les textes : types et prototypes, Paris, A. Colin.

- (2011b) : Genres de récit. Narrativité et généricité des textes, Bruxelles, Academia-L'Harmattan.

BRANCA-RosOFF, S. (1999) : «Types, modes et genres : entre langue et discours », Langage et société 87, 5-40.

BRASSAC, C. (2007) : «Co-responsabilité cognitive et dissolution de frontières », in P. Hert, M. Paul-Cavallier (éds), Sciences et frontières. Délimitations du savoir, objets et passages. Fernelmont (BE) : Éditions Modulaires Européennes \& InterCommunications, 159-176.

BRASSAC, C. et al. (2008a) : «Interweaving objects, gestures, and talk in context», Mind, Culture and Activity : An International Journal, 15(2), 208-233.

CARr, N., The Shallows: What the Internet Is Doing to Our Brains, [traduit en français par Internet rend-il bête ?], New York-London, W. W. Norton \& Company.

DE SAINT-GEORGES, I. (2012, sous presse) : « Nouvelles épistémologies en analyse du discours et des interactions : le paradigme de la Mediated Discourse Analysis », Semen 34.

HutChins, E. (1994 [1991]) : «Comment le cockpit se souvient de ses vitesses » (trad. de « How a Cockpit Remembers its Speed»), Sociologie du travail 4 : 461-473.

Kerbrat-ORECCHIONI, C. (2012) : «Le contexte revisité », CORELA-RJC Cotexte, contexte, situation, http://corela.edel.univ-poitiers.fr/index.php ?id=2627.

LABBÉ, H., MARCOCCIA, M. (2005) : «Communication numérique et continuité des genres : l'exemple du courrier électronique », Texto!, Septembre 2005, vol. $\mathrm{X}, \mathrm{n}^{\circ}$ 3. Disponible sur : http://www.revue-texto.net/Inedits/LabbeMarcoccia.html.

LAtour, B. (1997) : Nous n'avons jamais été modernes. Essais d'anthropologie symétrique, Paris, La découverte.

MAINGUENEAU, D. (2004) : «Retour sur une catégorie : le genre », dans Adam, J.-M. et al. (dir.), 2004, Texte et discours : catégories pour l'analyse, Dijon, Éditions universitaires de Dijon, 107-118.

MOIRAND, S. (2003) : «Quelles catégories descriptives pour la mise au jour des genres de discours ? », contribution à la journée d'étude « Les genres de l'oral », organisée par C. Kerbrat-Orecchioni et V. Traverso le 18 avril 2003, Université Lumière Lyon 2, http://gric.univ-lyon2.fr/ Equipe1/actes/journees_genre.htm. 
- (2006) : «Textes/Discours et Co(n)textes ; Entretiens avec J.-M. Adam, B. Combettes, D. Maingueneau, S. Moirand », Pratiques 129/130, 20-49.

Paveau, M.-A. (2006) : Les prédiscours. Sens, mémoire, cognition, Paris, Presses Sorbonne nouvelle.

— (2007) : «Discours et cognition. Les prédiscours entre cadres internes et environnement extérieur ", Corela (Cognition, représentation, langage), revue électronique : http://corela.edel.univ-poitiers.fr/document.php ?id=1672.

— (2009) : «Mais où est donc le sens ? Pour une linguistique symétrique », in Actes du deuxième colloque international Res per nomen. Reims : CIRLEP, 21-31.

- (2010) : «Une linguistique symétrique pour penser le discours », La pensée $d u$ discours [carnet de recherche], http://penseedudiscours.hypotheses.org/ $? \mathrm{p}=95$

- (2012a) : "Ce que disent les objets. Sens, affordance, cognition », in Quelle place pour les objets dans les sciences du langage et sciences de la communication? Synergies Pays Riverains de la Baltique, 9.

- (2012b) (dir.) (sous presse) : «Texte, discours, interaction. Nouvelles épistémologies », Semen 34.

- (2012c) (sous presse) : «Réalité et discursivité. D’autres dimensions pour la théorie du discours », Semen 34.

PAVEAU, M.-A., Rosier, L. (2010) : «Le discours des objets. Pratiques et techniques de circulation entre clandestinité et exhibition discursive ", in López Muñoz J.M. et al. (eds), La circulation des discours : médias, mémoire et croyances, Çédille. Revista des estudios francese, Monografias 1, http://dialnet.unirioja.es/servlet/articulo ?codigo=3399614, consulté le 23.10.2011.

PÊCHEUX, M., C. HAROCHE, P., HENRY (1971) : « La sémantique et la coupure saussurienne », Langages 24, «Épistémologie de la linguistique », 93-106.

RASTIER, F. (2001a, Arts et sciences du texte, Paris, PUF.

— $(2001 \mathrm{~b})$ : «Éléments de théorie des genres » (texte diffusé sur la liste fermée «Sémantique des textes », 2001), en ligne sur www.revue-texto.net.

SCHAEFFER, J.-M. (2007) : La fin de l'exception humaine, Paris, Gallimard.

SCOllon, R. \& S.W. SCOLlon (2003) : Discourses in Place: Language in the Material World. Londres : Routledge.

SCOllon, S. \& DE SAINT-GeORGES, I. (2012) : « Mediated Discourse Analysis », in J.P. Gee \& M. Handford (Eds,). The Routledge Handbook of Discourse Analysis. Londres \& New York : Routledge.

\section{Webographie}

Claved. Le rendez-vous hebdomadaire des édutwitteurs : http://claved.wordpress.com/2012/03/30/une-nouvelle-envolee-pour-le-claved/

Croisade, roman interactif de Thierry Crouzet écrit via Twitter : http://twiller.tcrouzet.com/the-end/ 
DOREJ, la page du wiki collectif décrivant l'expérience \#12hommes par Jean Dore : http://dorej.wikispaces.com/message/view/home/36229164

I-voix, le blog de jean-Marie Lebaut : http:/www.i-voix.net/

Enseignant en cherchant, un carnet de Delphine Regnard : http://enseignant.hypotheses.org/

Randonnée scripturale, le blog de Nathalie Couzon : http://randonnee.effetdesurprise.qc.ca/

Rough Time, le blog de Nicholas Carr : http://www.roughtype.com/

Technologies discursives, un carnet de Marie-Anne Paveau http://technodiscours.hypotheses.org/

Twittclasses francophones : http://twittclasses.posterous.com/

Laurent Zavack blog : http://laurentzavack.blogspot.fr/ 


\section{ANNEXE 1 \\ Tweetdoc de l'exercice \#conseilsorcière}

\section{\#conseilsorcière}

\section{\#conseilsorcière}

On doit écrire un petit conseil de sorcietre court, à la maniere de Jean

Tardieu. Jacky et Johanna

\section{Trending Words}

de, Giclasse_acou, à, les, l, rt, un, pas, ne, la, et, wous, suivez. absolument, c'est

\section{Event Participants}

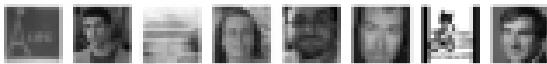

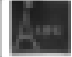

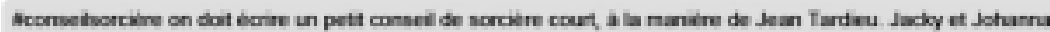

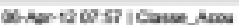

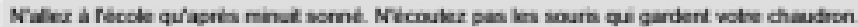

Aconselworcibre. Lisa

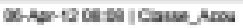

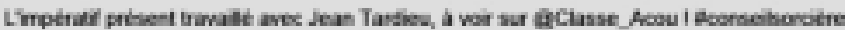

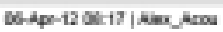

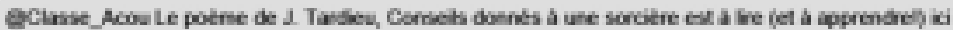

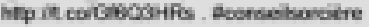

DS-Ape-120044 | Anex Acou

Je sub dans la GClasse_Acou et wob en anant-prenie leurs essab pour tmoeter des Acondeibonciere ateasing 4

Q6-12-121112!2vanas

Ne wous lavez pas anart wos 100 ans. Ne sonez pas de chez wous la nut. Mulissa acorbetsonotre.

06-10.12 12:3010anse Acou

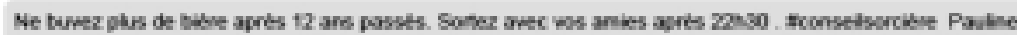

Q6-40.12 12.471 Canse Aewe

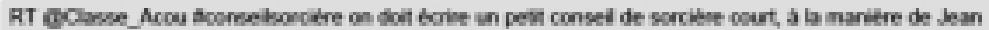
Tandieu. dacky et Joharna

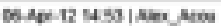

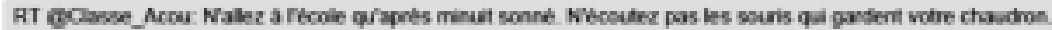
Aconsedsorcibre. Usa.

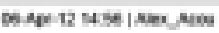

Ne cuarine vole soupe qua hut heures du natin

Naliex pas ae pete bois le scir.

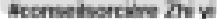


ANNEXE 2

Tweetdoc du \#claved du 23 mai 2012 - Page de présentation

\section{\#claved}

\section{Claved V2 - Classe $\mathbf{2 . 0}$}

rencontre claved du 23 mai

\section{Trending Words}

de, et, rt, classe, les, la, à, des, le, pour, un, du, en, (1)p_gagnon, une

\section{Event Participants}

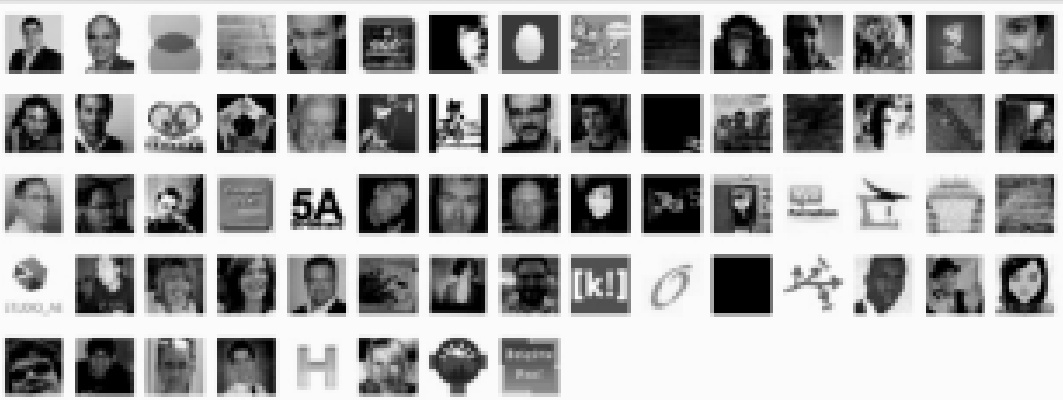

\section{Début du \#claved}

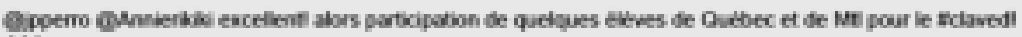
Ix)

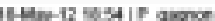

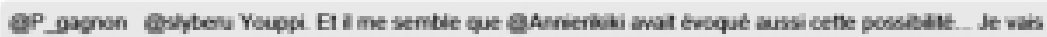
RT son message. Edaved

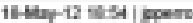

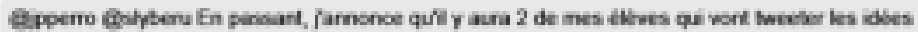
damenugement 20 lons du Aclawed I)

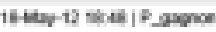

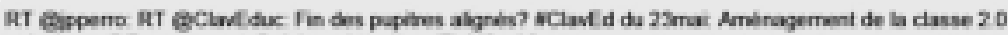

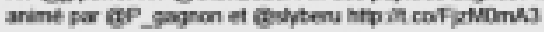

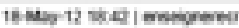

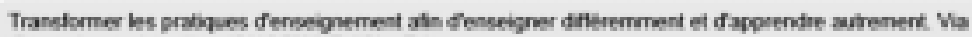
Gnoras ma hip it corceNCSWKF aClaved

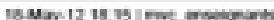

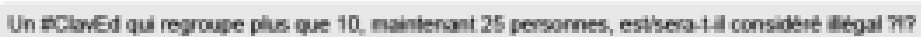

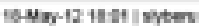

Fin des puptres bien abgnes? aClavEd du 23 ma Ameragement de la classe 20 anime par QP gagnon et

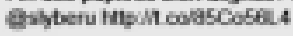

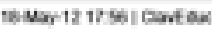

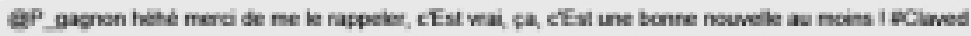




\section{Fin du \#claved}

Gsbberu Euh, faut righer paramkes a sco tweets (man). Actoned

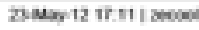

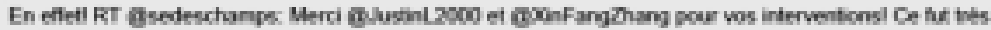
inbiessant de wous lnet aclaned

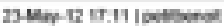

RT Gclaneduc: [ Jai u tedaved] Si vous kez cete dscussion, nerd de RT ce tweet (queston de starstiques]

$23 \mathrm{Nm}-1217: 41 / \mathrm{enc}$

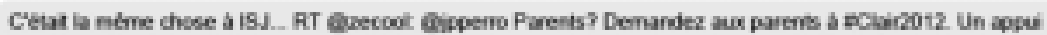

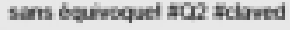

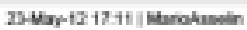

arecool je neptse, wive le copier-colber a la nain I Eclaved

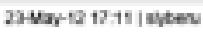

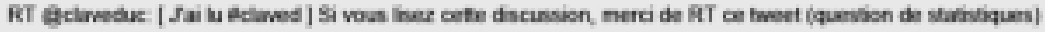

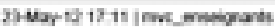

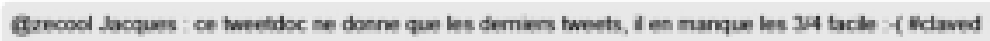

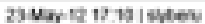

RT GClawEduk: [ Jai ha Aclaved ] Si vous lese cete dscusion, merd de RT ce tweet (quesbon de stabshques]

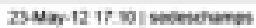

Merd a tous ceux qui onk porticipe a aclaved y Ca c'etat bien passet ?

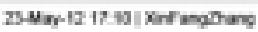

[ Jai iu aclaved ] Si vous lisez cethe dscussion, merci de RT ce tweet (question de statistiques] 